\title{
INSTRUMENTATION \& TECHNIQUES
}

\section{A microcomputer interface for decoding telemetry data and displaying them numerically and graphically in real time}

\author{
JOHN CIGAS \\ University of California, San Diego, La Jolla, California \\ and \\ A. PETER KLIMLEY \\ Scripps Institution of Oceanography, University of California, San Diego, La Jolla, California
}

\begin{abstract}
A microcomputer (IBM-PC) interface for decoding ultrasonic telemetry pulse-modulated signals from either unisensor or multisensor (time-multiplexed) transmitters is described. Pulse intervals, recorded by the interface, and corresponding independently measured parameter levels (i.e., swimming directions, speeds, depths, water temperature, and ambient irradiance levels) are entered into a calibration file. The IBM-PC uses this file to convert the signal's pulse intervals to sensor states and displays these in real time. States of up to eight multiplexed sensors can be displayed concurrently in either numerical or graphical format (as histograms) with low resolution (16 axis divisions) or, for a particular sensor, with high resolution (70 axis divisions). The circuit components mount on the IBM Prototype Card (with foil for interfacing logic) allowing easy installation in the IBM-PC's expansion slot. The $35 \mathrm{~K}$ of controlling software is written in Pascal with four subroutines in assembly language.
\end{abstract}

Describing the behavior patterns of mobile marine species (e.g., fishes, seals, dolphins, and whales) is a formidable task. The potential of ultrasonic telemetry transmitters for remotely identifying such patterns is great, especially when the number of sensors permitted in a transmitter is increased by the addition of a multiplexer (see Nelson, 1978). Methods of signal decoding, however, have not kept pace with transmitter development. Decoding is still most often done by timing intervals on a stop watch and then finding the corresponding sensor state on a calibration curve (for current reviews of such telemetry methodology see Harden-Jones \& Arnold, 1982, and Hawkins \& Urquhart, 1983). Only recently have a few investigators (Cunningham \& Peris, 1983; Rogers, Church, Weatherley, \& Pincock, 1984) begun to decode

This research was supported by contracts to the second author from the Office of Naval Research (N00014-83-KJ-0299) and NOAA (administered by the National Park Service; CX8000-4-0042) and by grants from the Foundation for Ocean Research, National Science Foundation (BNS84-19706), and National Geographic Society (2935-84). We would like to thank $T$. Johnson and J. Reeseman for help in designing the hardware and B. Benson for programming assistance.

John Cigas's mailing address is: Department of Electrical Engineering and Computer Sciences (C-014), University of California, San Diego, La Jolla, CA 92093. A. Peter Klimley's mailing address is: Bodega Marine Laboratory, University of California, Davis, P.O. Box 247, Bodega Bay, CA 94923. and store telemetry data using microprocessor-based systems. However, these are limited in their capabilities, processing only unisensor data and offering a single display of the data.

We describe a microcomputer (IBM-PC)-based telemetry decoder that: (1) processes a pulse-interval-coded signal into sensor states by interpolation or extrapolation based on a file containing tables of pulse intervals (PIs) and their corresponding behavioral and/or environmental measurements, (2) displays uni- and multisensor measurements in real time, (3) plots the states of up to eight sensors as histograms with low resolution, (4) plots measurements from a single sensor with high resolution, (5) prints out a hard copy of recently acquired data, and (6) stores measurements on a floppy disk. This data can then be rapidly searched with commercially available database software (e.g., dBASE II/III) for important but infrequent behavioral events, and these events as well as associated environmental factors can be studied in detail.

\section{ULTRASONIC TELEMETRY SYSTEM}

The resistive state of the sensor on the telemetry transmitter controls the time between successive ultrasonic pulses (in our case, ranging from $32-41 \mathrm{kHz}$ ). These pulses are converted by the receiver to either bursts of a $2-\mathrm{kHz}$ pure tone or changes in transistor-transistor logic 
(TTL) level. The decoder measures the intervals between these bursts or changes in logic level, and the resulting times (in millisecond counts) are then passed to the microprocessor (Intel 8088) of an IBM-PC for error checking, conversion from PIs to sensor levels, and display on a monitor before storage on a floppy disk. The decoder hardware mounts on a card that fits into an expansion slot of the IBM-PC. It should have a minimum of $128 \mathrm{~K}$ of memory and two $51 / 4$-in. disk drives, the first for the decoder's program and file with calibration values and the second for data storage. The system program is written in Pascal with four assembly language subroutines.

\section{Biotelemetry Device}

Signals from both uni- and multisensor transmitters can be decoded. The latter transmitter type uses an eightchannel multiplexer to alternate between sensors (for circuit design, see Nelson, 1978). A PI must be recognized as belonging to a particular sensor. This is accomplished by substituting a fixed resistor for a resistive sensor at a particular channel to produce a unique PI (a prompt in the program requests the channel's identity). This PI is recognized by the program and placed in the specified column on the screen with the next seven PIs, each corresponding to a particular sensor, placed in the following seven columns. It is necessary that the PIs produced by the sensors average greater than $300 \mathrm{msec}$, the PI required for the IBM-PC to complete its processing.

\section{Receiver-Microcomputer Interface Circuit}

The function of the interface circuit is to time the intervals between successive tone bursts or logic changes at the output of the telemetry receiver. The resulting measurements (in the form of millisecond counts) are passed to the microprocessor for further processing. The electronic components for the interface mount on the IBM Prototype Board, which has foil for the decoding logic and bus interface buffers already deposited on it (for schematic, see Figure 1).

Tone detection. On detection of a transmitter's signal, an ultrasonic telemetry receiver produces either a burst of a $2-\mathrm{kHz}$ sinusoidal wave at a phono output (Communications Associates, Inc. [CAI] $\mathrm{CS}-40$ and Dukane N15A235B) or a TTL square wave at a count output (CAI CS-40 and Ultrasonic Telemetry Systems MR1). In the former case, the signal burst is converted to TTL by a tone detector/phase-locked loop (Signetics, NE567N) (U8). It is tuned with a $2.7-\mathrm{k} \Omega$ resistor between pins 5 and 6 and a 200-pF capacitor at pin 6 for maximum sensitivity to $2 \mathrm{kHz}$, with $<3 \mathrm{~dB}$ attenuation of frequencies in a bandwidth $14 \%$ of the center frequency. On detection of a pulse within the configured bandwidth of U8, the tone detector's output drops from $5.0 \mathrm{~V}$ (logical 1) to $0.1 \mathrm{~V}$ (logical 0 ) for the duration of the tone burst. We found that pulses of a sinusoidal waveform at the input of U8 often produced chatter at its output, that is, a changing of the logical state at each successive rise in the sinusoidal waveform. This chatter was reduced in two ways: a $1.0-\mu \mathrm{F}$ capacitor was placed at pin 2 to act as a low-bandpass filter, and a feedback loop was formed by attaching the output of U8 at pin 8 to its input at pin 1 with a $10-\mathrm{k} \Omega$ resistor and $2.0-\mu \mathrm{F}$ capacitor. (For this and two other schemes for eliminating chatter at the output of U8, see Signetics Corporation, 1977, pp. 594-603.) Any residual chatter was filtered by configuring three NOR gates (Texas Instruments 74LSO2) (U6) at the output from U8 into a D latch, thus debouncing the output signal. For receivers producing a TTL square wave at their output, the tone detector/phase-locked loop and its associated circuitry can be bypassed.

Automatic gain adjustment. Even with the abovementioned precautions, strong input signals ( $>10 \mathrm{~V} \mathrm{p}$-p) caused a secondary 12-msec drop in the output of U8 following the initial drop on detection of an input pulse. For this reason, we added an automatic gain control to limit the level of the input signal U8. One half of the dual transconductance amplifier (U11) (National Semiconductor LM13600) was configured to produce an output signal of $1.2 \mathrm{~V}$ p-p. This output voltage can be adjusted by changing the resistance of the 1-M 2 potentiometer between pins 1 and 11 on $U 11$. The rate at which the output signal reaches the former level is controlled by a $25-\mathrm{k} \Omega$ potentiometer between pins 3 and 4 on U11.

Pulse interval timing. Interval measurement is accomplished by two of three timers in a programmable interval timer (Intel 8253-5) (U10). Both are down counters, programmed to decrement a loaded count (in our case, 10,000 ) by 1 for each incoming clock pulse. Each timer is enabled when its gate goes low and disabled when its gate goes high. When the latter occurs, the attached interrupt request line also goes high, momentarily changing the priority of operations of the IBM-PC's microprocessor so that it services an interrupt. This is accomplished by sending a control byte to the counter to stop counting and then reading the count. Because the timer decrements its loaded value, the frozen count must be subtracted from the loaded value (in our case, 10,000) to obtain the PI. The PI is then stored in a 512-word ring buffer, a FIFO queue with its head connected to its tail (Standish, 1980).

The timers are alternately enabled by the complementary $\mathbf{Q}$ and $\mathbf{Q}^{\prime}$ outputs of a D flip-flop (Texas Instruments 74LS74) (U9). The input to U9 is its own $Q^{\prime}$ output, causing the two outputs to change logical state on every clock pulse. The output from the tone decoder acts as the clock. Thus, every TTL square wave detected effects a change in the states of U9's outputs, which in turn stop one counter and start the other. For instance, when $Q$ goes low, timer 0 begins decrementing, and when $Q$ goes high, it stops decrementing. At the same time $\mathbf{Q}$ goes high, $\mathrm{Q}^{\prime}$ becomes low, and timer 1 starts to decrement. The interrupts are also alternated. When $Q$ goes high, the interrupt for timer $\mathbf{0}$ is serviced, and the resulting PI is placed in the buffer. When $Q^{\prime}$ goes high an interrupt is serviced 


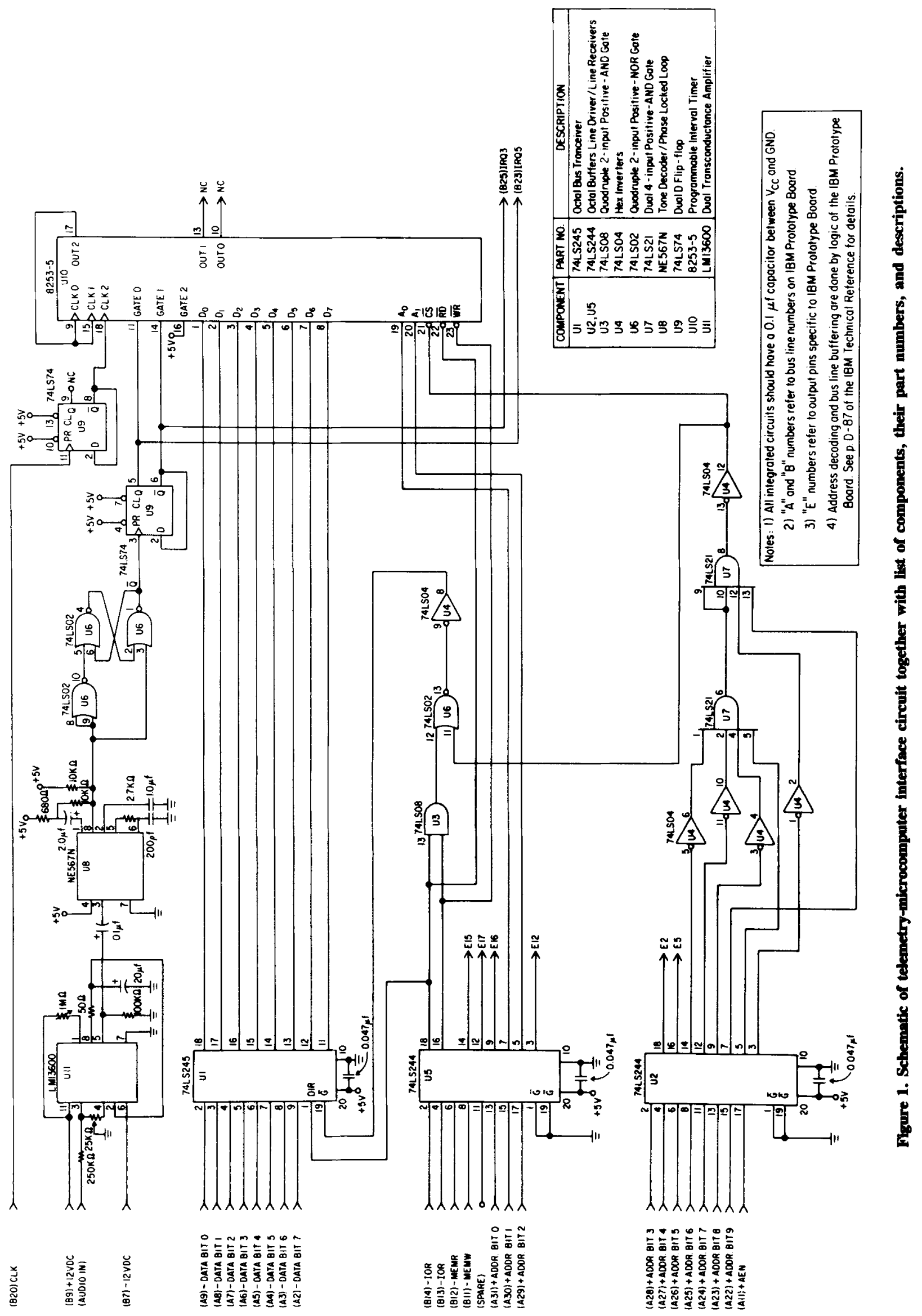


for timer 1, and that PI is stored in the buffer. The two interrupt service routines are identical except for the timer address to which each reads and writes.

The programmable interrupt controller (Intel 8259a) on the IBM-PC system board must be reprogrammed to accept interrupts from the decoder. The controller prioritizes interrupt requests from peripheral devices (e.g., keyboard, printer, and disk drives), and presents interrupts to the IBM-PC's microprocessor one at a time. A software mask is written to selectively enable channels IRQ3 and IRQ5 of the controller, allowing them to recognize the interrupt signals on B23 and B25 of the bus. A second mask is required to disable these two channels on termination of the program, reestablishing the former priority of requests.

To obtain interval measurements in milliseconds, the output from timer 2 serves as the clock input to timers 0 and 1 . Timer 2 is programmed to generate a $1-\mathbf{k H z}$ ( $1-$ msec period) square wave with an input clock rate of $2.385 \mathrm{MHz}$. The output from timer 2 is connected to the clock inputs of timers 0 and 1 , thus decrementing their loaded counts by milliseconds. The $4.77-\mathrm{MHz}$ frequency of the IBM-PC's system clock must be halved to be within the acceptable range of U10. This is done by the second D flip-flop on U9.

\section{Programming}

Software (software and manual are available from the second author) was written in both MT +86 Pascal and ASMT-86 Assembly Language (Version 3.1) from Digital Research. The use of this version of Pascal allowed not only the menus and displays, but also the routines for handling interrupts and selecting the operating modes of the peripheral processors, to be written in high level computer language. We needed only four assembly language routines, the first and second for repairing a defect in the compiler-generated code, the third for allowing input to the program while recording tracking data, and the fourth for selectively scrolling the screen. The first procedure saves the current data segment in the code segment register at the beginning of the program, and the second restores that segment to the code segment at the beginning of each interrupt handler. The fourth procedure, used with the graphical displays, scrolls only the lower 21 lines of the screen while leaving the first 3 lines with the header in place. The executable program is $35 \mathrm{~K}$ long and is controlled through a hierarchy of menus (Figure 2).

Telemetry signal verification. In order to distinguish the order in which the sensors are alternated by the eightchannel multiplexer, a fixed resistor is placed at the input to one channel to provide a reference interval. The

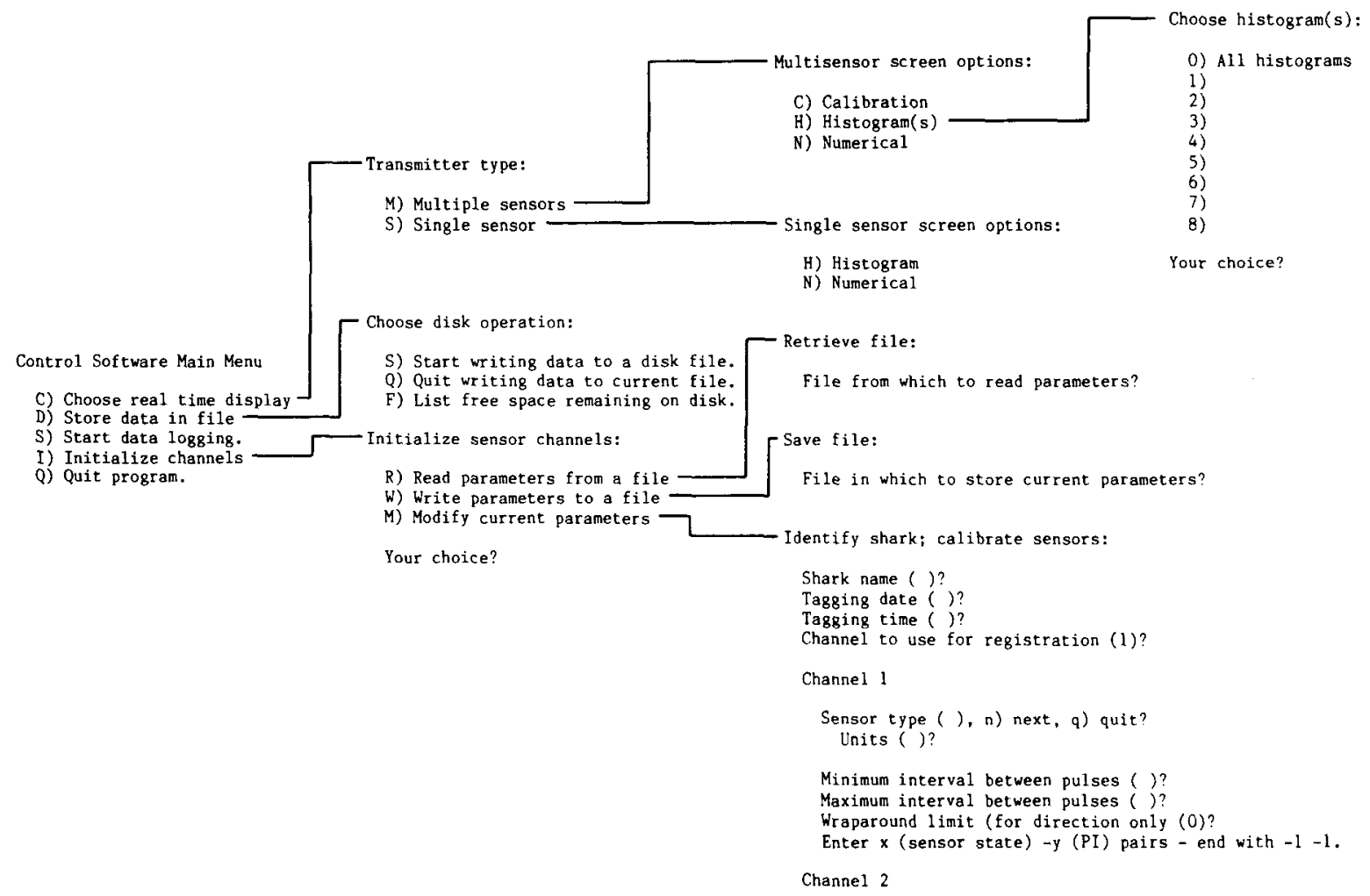

Figure 2. Hierarchical diagram of software menus. 
decoder's software searches through the ring buffer for a PI of this value, and on detection displays it in the appropriate column of the display. The following sensor state is displayed in the next column, and those from the remaining six channels in the following six columns; then this process is repeated. One further check of the signal is necessary. The telemetry receiver might not detect a pulse of the transmitter's signal if the subject of the tracking were to momentarily exceed the receiver's signal detection range; or the receiver might mistake a pulse of ambient noise (such as the broadband clicking of snapping shrimp) for a pulse from the transmitter. These errors would disturb the sequence in which the PIs were received and result in their being placed into improper columns. To detect such errors, the progam (1) searches for a reference PI within a specified range, (2) looks for the next value within that range, (3) counts the intervening PIs, and (4) compares that count to the number of channels in the multiplexer (in our case, eight). If the count is the same as the channel total, the PIs are passed to another part of the program for further processing; if not, the values are discarded and the program searches again for the reference value. A message is displayed on the monitor at this time to alert the user to the telemetry signal's deficiency.

Conversion of pulse interval to sensor state. The PIs for each of the sensors must be converted into their corresponding sensor states. For this purpose, an initialization file is made for each tagged shark containing a description of the tracking (shark's identity, tagging date and time) and sensor calibrations for up to eight channels. This file is retrieved from the system disk whenever an investigator wants to track that particular shark. Calibration values consist of PIs, measured by the decoder using its calibration mode (Figure 3A), and independently measured sensory states (e.g., swimming directions, speeds, depths, water temperatures, and irradiance levels). The two columns of values are entered row by row (Figure 3B). The program then determines the sensor state corresponding to a PI based on linear interpolation for $i_{1}<i_{s}<i_{2}$

$$
s_{s}=s_{1}+\frac{\left[\left(i_{s}-i_{1}\right)\left(s_{2}-s_{1}\right)\right]}{\left(i_{2}-i_{1}\right)},
$$

where $i_{1}=$ shorter PI in file, $s_{1}=$ corresponding sensor state, $i_{2}=$ longer PI, $s_{2}=$ sensor state; $i_{s}=$ PI from telemetry signal, or on extrapolation for $i_{s}>i_{b}$ :

$$
s_{s}=s_{b-1}+\frac{\left[\left(i_{s}-i_{b-1}\right)\left(s_{b}-s_{b-1}\right)\right]}{\left(i_{b}-i_{b-1}\right)},
$$

where $i_{b}=$ upper boundary PI, $s_{b}=$ corresponding sensor state, $i_{b-1}=$ nearest $\mathrm{PI}$, and $s_{b-1}=$ sensor state. For $i_{s}<$ lower boundary, extrapolation is based on a formula similar to the latter.

Displays. The decoder possesses three real-time display modes: calibration, numerical, and graphical. In the calibration mode, PIs (in milliseconds) are displayed on
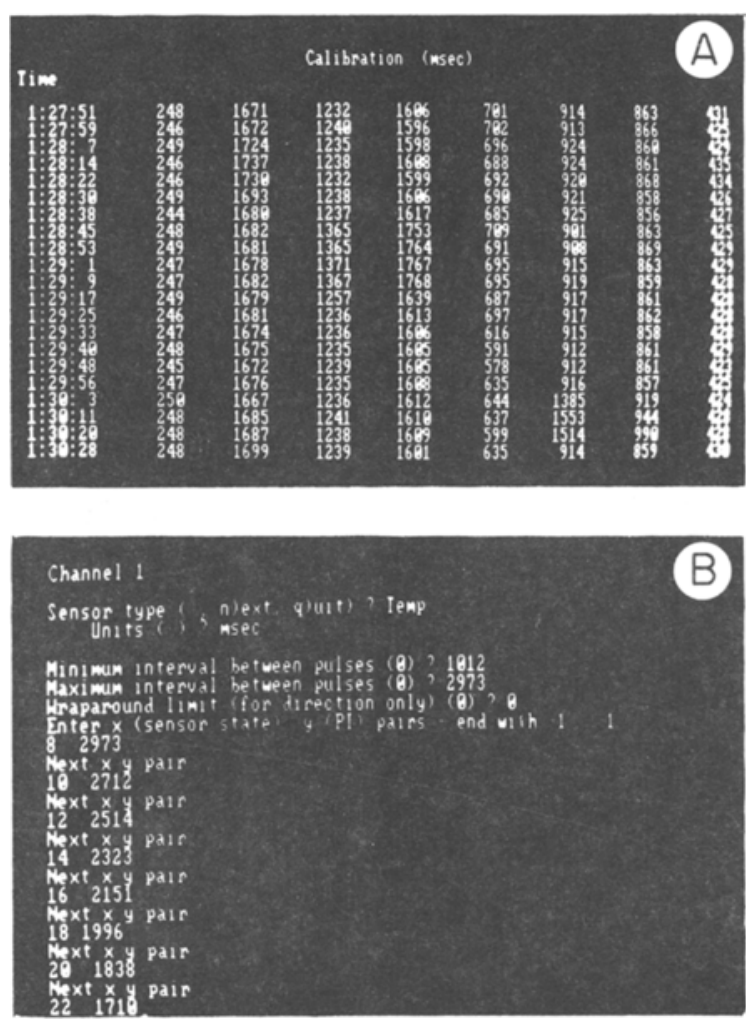

Figure 3. Intervals (in milliseconds) between successive tone bursts from the transmitters can be measured and displayed on the monitor in the calibration mode (A). The intervals and corresponding sensor levels (temperature, in this case) are entered into an initialization file (B). When decoding directional data such as swimming direction, the wraparound limit must be set at 360 .

the monitor (see Figure 3A). Following a header in the first three rows identifying the decoder's mode and those units measured, time since track start (in hours:minutes:seconds) is given, followed by eight PIs. The cursor then returns to the next row, updates the time in the first column, and prints PIs in the next eight columns.

In the numerical mode, an integer or real number $(i<$ 40) is displayed. For a unisensor transmitter (Figure 4A), each sensor state value is preceded by a time update; for a multisensor transmitter (Figure 4C), states of up to seven sensors (for the eight channels of the multiplexer) are printed together with the registration PI on each row.

In the graphical mode, sensor states are presented in histograms (with bars oriented horizontally). The histogram displayed for a unisensor transmitter (Figure 4B) has a horizontal axis with 70 divisions, separated into seven classes of 10 divisions each. The value of a single increment is displayed above the graph, and values for the size classes are printed immediately above the class ticks. There are two options in the multisensor mode. First, up to eight vertical bar graphs, one for each channel of the multiplexer, are displayed together on the monitor (Figure 4D). Each graph is labeled with sensor type 

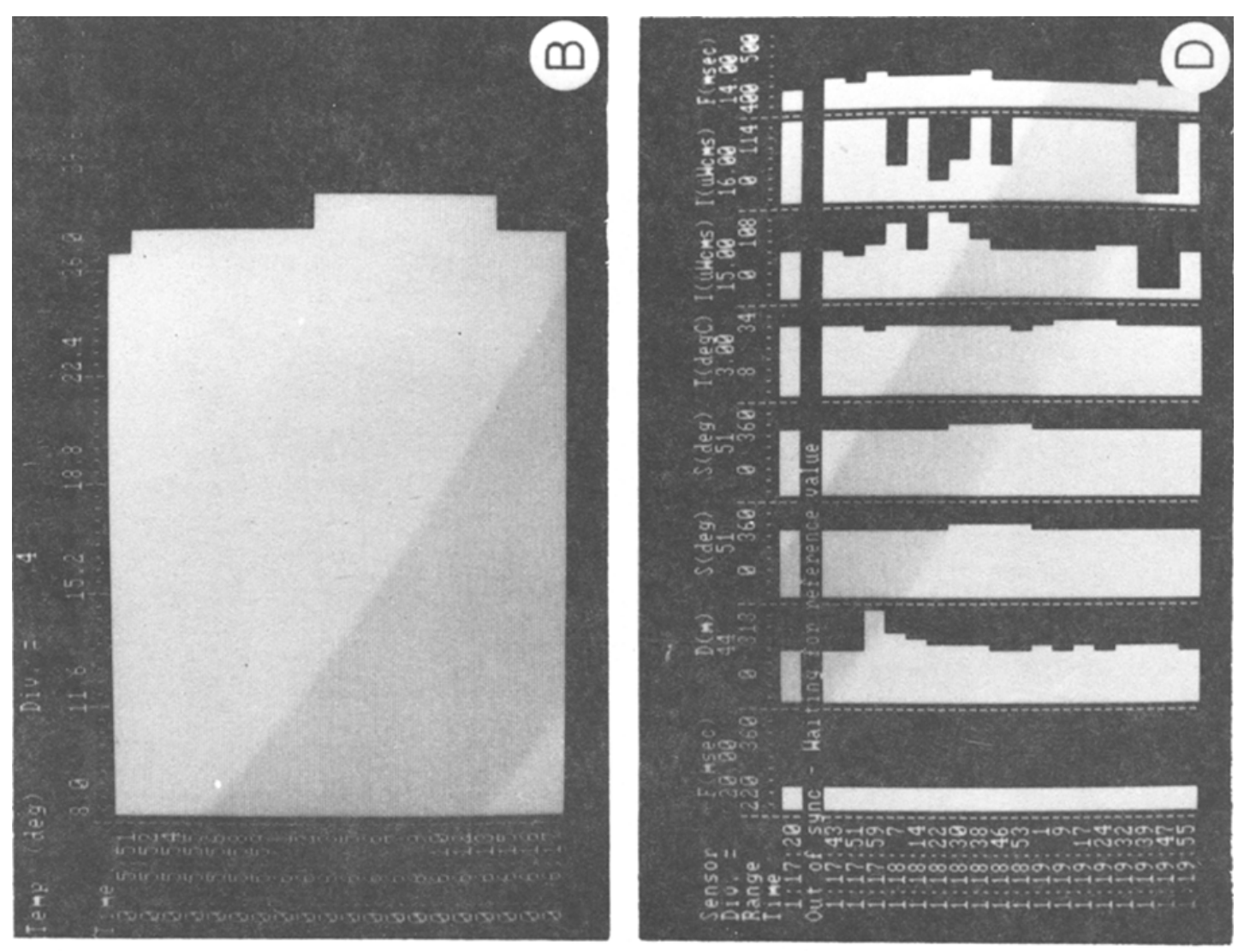

寞当

3.

家战

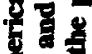

可.9

8

8

을

$8<$

常

急

\&

동

重

量.

뭉

于

$\frac{1}{8}$

远

불

8

g

害量

¿

ซึ่

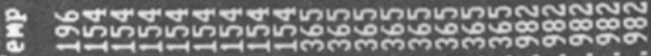

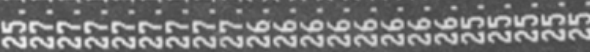

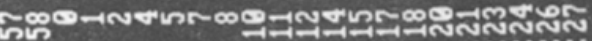
r. $x$ ะอง 

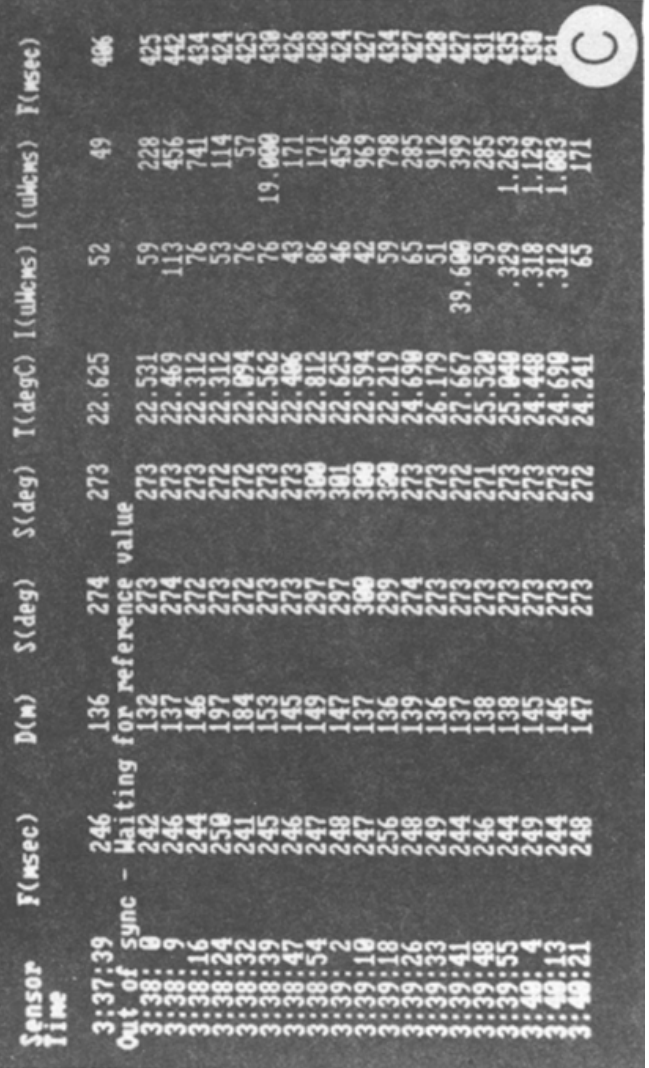


and units. Each graph consists of a horizontal axis with 16 divisions. The lower and upper ranges of the scale are printed above the ends of each axis; the increment for each division is given above the center of each axis. The second option provides a higher resolution histogram similar to that displayed for a single sensor transmitter.

Data storage. Sensor values are stored in an ASCII text file in the record format either on a second disk drive (the system's program and calibration file are on the first disk drive) or a hard disk drive. A record consists of either nine fields (the time since system start-up and eight PIs [calibration mode] or a registration PI and seven sensor states [multisensor mode]) or two fields (the time prompt and a single sensor state [unisensor mode]). With a mean sensor PI of $1 \mathrm{sec}$, about $18 \mathrm{~h}$ of continuously transmitted multisensor data can be recorded on a 51/4-in. double density floppy disk. Storage space (ca. 40K) is reserved on the data storage disk for the dBASE program. This progam must be present to convert the files, which are written to the disk from the interface in ASCII format, to dBASE II/III format. When only $80 \mathrm{~K}$ of disk space remain free for data storage, a warning is displayed on the monitor each time a write is carried out by the IBM-PC. The telemetry program also allows the tracker to check what space remains free on a second disk drive. The files can be modified with a text editor, such as WORDSTAR, or analyzed with database management software, such as dBASE II/III. The latter software allows the user to search the file for sensor states occurring at specified times in order to study diel rhythms in behavioral activity. Additionally, an infrequent behavioral event (e.g., predation) may be found quickly in very long files. The file can be queried for sensor states (e.g., rapid swimming speeds and deep or shallow swimming depths) indicative of such an event.

\section{CONCLUSIONS}

The microprocessor-based telemetry decoder described here provides a means of rapidly and continuously gathering behavioral data for large mobile marine species. The decoder displays this data in real time either numerically or graphically. Experimental manipulations can be carried out, and the species' responses to such manipulations can be monitored concurrently. Furthermore, by searching large time blocks of such fine-scale behavioral description with database management software, the investigator can study infrequent yet important behaviors (e.g., predation and courtship) in large mobile oceanic species such as the tunas, billfishes, sharks, dolphins, and whales.

\section{REFERENCES}

Cunningham, C. L., \& Peris, J. (1983). A microcomputer system for temperature biotelemetry. Behavior Research Methods \& Instrumentation, 15, 598-603.

HARDEn-Jones, F. R., ARNold, G. P. (1982). Acoustic telemetry and the marine fisheries. Symposia, Zoological Society of London, 49, 75-93.

Hawkins, A. D., Urquhart, G. G. (1983). Tracking fish at sea. In H. E. MacDonald \& I. G. Priede (Eds.), Experimental biology at sea (pp. 103-166). London: Academic Press.

Nelson, D. R. (1978). Telemetering techniques for the study of freeranging sharks. In C.S. Hodgson \& R. F. Mathewson (Eds.), Sensory biology of sharks, skates, and rays (pp. 419-482). Arlington, VA: Office of Naval Research.

Rogers, S. C., Church, D. W., Weatherley, A. H., \&incock, D. G. (1984). An automated ultrasonic telemetry system for the assessment of locomotor activity in free-ranging rainbow trout, Salmo gairdneri, Richardson. Journal of Fish Biology, 25, 697-710.

SigNeTICs CoRPORATION. (1977). Analog specifications, applications, military summary. Sunnyvale, CA: Author.

Standish, T. A. (1980). Data structure techniques. Menlo Park, CA: Addison-Wesley.

(Manuscript received June 28, 1985;

revision accepted for publication November 4,1986 .) 